IAU Colloquium 164: Radio Emission from Galactic and Extragalactic Compact Sources

ASP Conference Series, Vol. 144, 1998

J. A. Zensus, G. B. Taylor, \& J. M. Wrobel (eds.)

\title{
The VSOP Mission-A New Era for VLBI
}

H. Hirabayashi

ISAS, 3-1-1 Yoshinodai, Sagamihara, Kanagawa 229, Japan

Abstract. The first VSOP satellite, HALCA, was successfully launched on 12 February 1997, ushering in a new era for VLBI astronomy. This paper will give a brief overview of the mission, satellite and the on-going In-Orbit Checkout of the mission elements.

\section{The VLBI Space Observatory Program}

The VSOP (VLBI Space Observatory Program) mission is led by the Institute of Space and Astronautical Science (ISAS), in close collaboration with the National Astronomical Observatory (NAO) of Japan. NASA/JPL, NRAO and CSA are key collaborating institutions, and most radio-telescopes world-wide, including the VLBA, EVN and AT, will participate in VSOP observations.

Funding for development of the VSOP satellite, MUSES-B, was first received in April 1989 in the first year of the Heisei era in Japan. Because of many new technologies needed for space-VLBI, ISAS selected the satellite as an engineering experiment, named MUSES, for the Mu-series Space Engineering Satellite. After the completion of engineering tests, the spacecraft is handed to the scientific community for space-VLBI observations. With the great efforts of scientists and with the great partnership of international colleagues, the VSOP mission has evolved into more of a scientific mission.

The 8 meter diameter radio-telescope in an elliptical Earth orbit will make the first dedicated space-VLBI observations on baselines up to $30,000 \mathrm{~km}$. Due to the Earth's oblateness the longitude of the ascending node and argument of perigee precess, and so good $(u, v)$ coverage over the whole sky will be obtainable for at least some part of the (nominally three year) life of the mission. The observing frequencies are $1.6,5$ and $22 \mathrm{GHz}$, with data down-loaded in real-time on a $128 \mathrm{Mbps}$ link supported by a dedicated five station telemetry network (Hirabayashi et al. 1992; Hirosawa \& Hirabayashi 1995). The 10-station VSOP correlator at Mitaka, 20-station VLBA correlator at Socorro, and 6-station S2 correlator at Penticton will be used for correlation. The JIVE correlator could be used in the latter part of the mission when it has been completed.

On baselines to the same ground antenna, VSOP will have, at 1.6 and $5 \mathrm{GHz}$, sensitivity a factor of about 6 better than that of TDRSS-OVLBI at $2.3 \mathrm{GHz}$ (including the improvement from the larger VSOP bandwidth). The sensitivity of a baseline between the satellite and a VLBA antenna will be a factor of $\sim 6$ worse than between two VLBA antennas. Because of the limited sensitivity, most of the observations will be limited to high brightness sources. Nearly all continuum observations will be of active galactic nuclei (AGNs) with some exceptions for possible flare stars, galactic jet sources, or supernovae events. For baselines between the satellite and VLBA antenna, the estimated number of detectable sources will be $1000-2000$ at $5 \mathrm{GHz}$.

Mapping periods of 2-4 orbits will be typical for VSOP imaging observations, to build up the $(u, v)$-coverage for good imaging capability. The high resolution and high dynamic range imaging capability are the most important 
features of the VSOP mission, which differs from the Radioastron project which aims more for resolution with the sacrifice of image quality. Observations with 1-2 orbits will be used for survey observations or model-fitting type observations. Observations are classified into two categories; the peer reviewed General Observing Time Program and the mission-led Survey Program. The Survey Program will deal with large statistical sample, to be observed with a smaller number of ground telescopes.

Monitoring of structure changes will be to some degree limited by the changing $(u, v)$-coverage. Both the dynamic range and the synthesized beam shape will vary with observing epoch due to the orbital precession.

\section{The VSOP Satellite, Orbit, Launch, and Tracking Support}

The spacecraft is 3 -axis stabilized and weighs $830 \mathrm{~kg}$ (including the reaction control system fuel). The main reflector of the satellite is a deployable parabola composed of a gold-coated molybdenum mesh with an $8 \mathrm{~m}$ diameter reflector, and a $0.5 \mathrm{~mm}$ rms surface accuracy expected. The multi-waveband feed horn is located at the Cassegrain focus with left-hand circular polarization receivers. The observing bands $-1.60-1.73 \mathrm{GHz}, 4.7-5.0 \mathrm{GHz}$ and $22.0-22.3 \mathrm{GHz}$ - include standard VLBI continuum frequencies and the main $\mathrm{OH}(1.6 \mathrm{GHz}$ band) and $\mathrm{H}_{2} \mathrm{O}(22 \mathrm{GHz}$ band) maser lines.

The final integration testing of the spacecraft finished at ISAS, Sagamihara, at the end of 1996, and the satellite was then moved to the Kagoshima Space Center for launch.

The MUSES-B satellite was the first launch with the new ISAS M-V rocket. The expected apogee and perigee heights were $19500(+4000 /-3000) \mathrm{km}$ and $1000 \mathrm{~km}$ respectively, with an orbital period of about 5.9 hours. As MUSES-B was the first launch of the $M-V$ rocket, the uncertainties were quite large.

The satellite was launched from the Kagoshima Space Center on 12 February 1997 . The satellite was successfully placed in an elliptical orbit with an apogee height of $21,250 \mathrm{~km}$, a perigee height of $220 \mathrm{~km}$, and an inclination angle of $31^{\circ}$. HALCA's first pass over the Kagoshima Space Center occurred 6.5 hours after launch. During the pass it was confirmed that the solar paddles and Ku-band antenna had deployed correctly.

After launch the satellite Muses-B was renamed HALCA, the Highly Advanced Laboratory for Communications and Astronomy. In Japanese, HALCA (pronounced Haruka) means "far away". This was 10 and half years since the first successful space VLBI demonstration, the TDRSS-OVLBI experiment (Tracking and Data Relay Satellite System-Orbiting VLBI Experiment, 19861988 - Levy et al. 1986).

Between 14 and 20 February three perigee raising maneuvers were conducted, increasing the perigee height to $560 \mathrm{~km}$. This is the observing orbit with the orbital period of 6 hours and 20 minutes.

The VSOP satellite is commanded at Sagamihara Operations Center at ISAS once a day by $2.1 \mathrm{GHz}$ telemetry through the Kagoshima Space Center. For VLBI observations the satellite needs special real-time telemetry support. The satellite will receive uplink from the hydrogen-maser-based frequency standard at $15.3 \mathrm{GHz}$, and will down-link data at $14.2 \mathrm{GHz}$ (at $128 \mathrm{Mbps}$ ) in real time for 
recording. Five $15 \mathrm{GHz}$-band tracking stations are employed: at Usuda (Japan), operated by ISAS; Green Bank (USA), operated by NRAO; and Tidbinbilla (Australia), Goldstone (USA), and Madrid (Spain), operated by NASA/JPL. This ground telemetry network will also play a key role in orbit determination for correlation.

\section{In-Orbit Checkout}

HALCA's sub-reflector was deployed on February 24th. The $110 \mathrm{~cm}$ diameter, hexagonal sub-reflector is supported on three legs, each of which are hinged in three places. Deployment took only a matter of minutes, and the sub-reflector is now in position $3.4 \mathrm{~m}$ above the main antenna surface.

The deployment of the main antenna took place during tracking passes on the 27th and 28th of February. The six booms were extended $4.8 \mathrm{~m}$, and tensioners then extended an additional $30 \mathrm{~cm}$ to deploy the gold-coated molybdenum mesh. This was one of the most important events after launch.

The first part of the in-orbit checkout began with bus-system checks, attitude control system, and so on. This has gradually shifted to radioastronomy system checks.

The first tests of the $15 \mathrm{GHz}$ telemetry link between the Usuda tracking station and HALCA took place during the second week of March. Astonishingly, the link with $128 \mathrm{Mbps}$ was established in the first pass. This was a critical path for the mission, because this was the single route without redundancy. Since then the other real-time telemetry stations have been slowly brought on-line. Before the launch, an extensive series of compatibility tests among the three differentlydesigned tracking stations (Usuda, Green Bank and DSN) were carried out.

The system temperatures of 1.6 and $5 \mathrm{GHz}$ receivers are better than expected, while the $22 \mathrm{GHz}$ receiver is worse. $\mathrm{OH}$ maser lines at $1.6 \mathrm{GHz}$ band were detected, and by comparison with $64 \mathrm{~m}$ antenna, we have $50 \%$ aperture efficiency with $5 \%$ accuracy, which is in good agreement with the expected nominal value of $48 \%$. By this fact, we are confident that antenna is deployed successfully with reasonable shape. By comparing the spectral shape to those from the $64 \mathrm{~m}$ spectra of RCP and LCP, we could also confirm that HALCA is receiving LCP as designed.

In April HALCA entered its first series of eclipses, with these occurring during the long apogee passes. Radioastronomy checkouts and experiments were very much limited during this time.

In-orbit checkout (IOC), including checks of the telemetry antennas and radiotelescopes, is in progress in partnership with the international operational elements. The IOC phase is a very complicated one. Numerous interactions are taking place among the operational elements. After the detection of fringes between HALCA-ground antennas, the space-VLBI world will become transparent to us. After the Big Bang of M-V launch on February 12, the international team is collaborating so that the tiny HALCA (electron) will be combined with the earth (proton).

Soon after the Socorro meeting, on May 7th, the first successful interferometric tests between the satellite and a ground radio telescope were conducted with HALCA and the Usuda $64 \mathrm{~m}$ telescope. Observations of the bright, com- 
pact quasar PKS 1519-273 at $1.6 \mathrm{GHz}$ were made. Data from the satellite were down-linked to the Usuda $10 \mathrm{~m}$ tracking station. The data were correlated at the Mitaka correlator, and first fringes were found on 13th May.

Fringes to HALCA were found in a second $1.6 \mathrm{GHz}$ experiment using the Usuda $64 \mathrm{~m}$ telescope, the Kashima (CRL) $34 \mathrm{~m}$ telescope and HALCA, on May 13th. Fringes were found on all three baselines, allowing the "closure phase" to be obtained. This was found to be stable and distributed around zero, as expected. This is another important step towards full space VLBI observations!

\section{Ground Radio Telescopes and Correlators}

A number of ground radio telescopes (GRTs) have committed time for simultaneous observations with VSOP. Commitments of ground radio telescope time for the mission have been negotiated through the Global VLBI Working Group (GVWG), formed under URSI Commission J. Participating VLBI networks include the Very Long Baseline Array (VLBA) and the European VLBI Network (EVN), APT, DSN antennas, and individual telescopes.

In order to test the various interfaces between the VSOG (VSOP Science Operations Group) and participating ground radio telescopes, the arrays and other telescopes agreed to participate in a series of VSOP test runs, which started in February 1996 and continued until launch. Observations of bright continuum and line sources have been made in a variety of formats. Both hardware verification and information exchanges, from schedule files through to observation logs and correlator input logs were tested. The GVWGtwg (technical working group) was established for these activities.

The standard VSOP recording mode has two channels of $16 \mathrm{MHz}$ bandwidth with 2-bit sampling, giving a $128 \mathrm{Mbps}$ down-link data stream. The recording format of VLBI data is dependent upon the ground facilities involved. Three different tape formats are used: VSOP, VLBA/MkIV, and S2. Depending on the participating GRTs, VSOP observations will be correlated at the VSOP correlator in Mitaka, Tokyo (first fringes seen September 1995), the VLBA correlator in Socorro, the S2 correlator in Canada (first fringes April 1995), or the JIVE MkIV correlator in Europe (scheduled for completion in 1998).

Experiments involving two or more formats will require conversion of some tapes at Mitaka, which will have two S2/VSOP and two VLBA/VSOP bidirectional copiers. Canadian involvement in the VSOP mission with S2 data acquisition terminals distributed worldwide and the operation of the Penticton correlator has also greatly eased compatibility problems.

The $64 \mathrm{~m}$ antenna at Usuda will participate as a radiotelescope. A near real-time correlator is located at Usuda for fringe verification between the $64 \mathrm{~m}$ antenna and the Muses- $B$ antenna (via the Usuda telemetry station). In addition, the Muses-B $8 \mathrm{~m}$ antenna pointing can be calibrated by observing maser sources and auto-correlating the data received by the Usuda telemetry station with the digital correlator at Usuda. A wide band optical fiber link is available now on loan from NTT for real time connection among the four important operational elements for VSOP in Japan: Usuda, Nobeyama, Sagamihara and Mitaka. Real-time monitoring of the spectrum or real-time recording at Sagamihara or at Mitaka of signals down-linked from HALCA via the Usuda telemetry station, or 
from the telescopes at Usuda or Nobeyama is possible. For this experiment both ISAS and the NAO VSOP team are funded from Tsushin-Hoso-Kiko. Currently, monitoring at Sagamihara operations center for real-time spectral monitoring of the HALCA video-band is operational. This is effectively used for $8 \mathrm{~m}$ antenna pointing calibrations using maser lines.

\section{Science Operation of VSOP, and Further Information}

The mission's science operations are led by the VSOP Science Operations Group (VSOG), centered at ISAS with its core members from ISAS and NAO. The scientific policy is determined by VSOP International Science Council (VISC), members of which were selected from the world-wide community.

General Observing Time is open to proposals from astronomers worldwide, and the proposals are reviewed based on their scientific merits by an international Science Review Committee, and scheduled taking into account the resource availability. The first Announcement of Opportunity was issued in June 1995 for the first 17 months from May 1997. One hundred and fifty proposals were received, ranked, and scheduling is currently being done.

The VSOP Announcement of Opportunity and Proposer's Guide provide a detailed description of the mission components and activities. Interested readers are encouraged to refer to these documents, which are available from the VSOP anonymous-ftp site, ftp.vsop.isas.ac.jp (133.74.2.131), in the /pub directory.

The VSOP World Wide Web site (http://www.vsop.isas.ac.jp/) contains additional information and pictures of mission activities. Regular updates, the VSOP Newsletter, are circulated electronically every two weeks.

After the successful launch, deployment of the $8 \mathrm{~m}$ antenna, functioning of the on-board modules, linkage with telemetry stations, and subsequent fringe detection with the ground radio telescopes, we are becoming more confident that the VSOP mission will start a new era. It is obvious that we need a successor to this mission. And, I am pleased to report that ISAS now has formed a Working Group responsible for proposing the next Space-VLBI mission. I hope this is not the last, and I look forward to future missions to continue exploring the universe.

Acknowledgments. The author thanks Dr. Philip Edwards for kind kelp in preparing this paper for its launch.

\section{References}

Hirabayashi, H., et al. 1992. Proc. 18th ISTS (Kagoshima), 1813-1820.

Hirosawa, H., \& Hirabayashi, H. 1995. IEEE AES Systems Magazine, 10, No. 6, 17-23.

Levy, G. S., et al. 1986. Science, 234, 187-189. 